\title{
Letter
}

Nadine Andric ${ }^{a, \star}$, Tobias Ehmke ${ }^{a}$, Nico S. Stumpp, Tammo Ripken, Alexander Heisterkamp and Meike Stiesch

\section{Nonlinear laser scanning microscopy of oral multispecies-biofilms: fixative induced fluorescence as a fast and economical in vitro screening method}

DOI 10.1515/bnm-2015-0028

Received December 30, 2015; accepted March 15, 2016; previously published online April 18, 2016

\begin{abstract}
In this letter we report a fast and easy method which could be used for initial screening of multispeciesbiofilm development on putative new dental implant materials. Most staining methods require numerous washing steps that can result in detachment of loosely bound biofilms and therefore falsify the results. Thus, we used glutaraldehyde fixation, which induces autofluorescence through bacterial membrane protein cross-linking and concurrently stabilizes the biofilm structure. We analyzed the biofilms with nonlinear laser scanning microscopy and were able to (I) evaluate the multispecies-biofilm growth and (II) distinguish between bacterial species based on different two-photon autofluorescence intensities.
\end{abstract}

Keywords: autofluorescence; dental implant; glutaraldehyde; multiphoton microscopy; periimplantitis; Schiff base.

Multispecies-biofilms play an important role in the development and progression of periimplantitis, which is

a Nadine Andric and Tobias Ehmke: These authors contributed equally to this work.

*Corresponding author: Nadine Andric, Department of Prosthetic Dentistry and Biomedical Materials Science, Hannover Medical School, 30625 Hannover, Germany, E-mail: andric.nadine@mh-hannover.de Nico S. Stumpp and Meike Stiesch: Department of Prosthetic Dentistry and Biomedical Materials Science, Hannover Medical School, Hannover, Germany

Tobias Ehmke and Tammo Ripken: Department of Biomedical Optics, Laser Zentrum Hannover e.V., Hannover, Germany Alexander Heisterkamp: Department of Biomedical Optics, Laser Zentrum Hannover e.V., Hannover, Germany; and Institute of Quantum Optics, Leibniz University of Hanover, Hannover, Germany considered as one of the major complications in dental implantology. If left untreated, the bacterial induced inflammation leads to bone loss and in the end to implant failure $[1,2]$. To improve the clinical situation, current research efforts focus on the development of new implant materials with antimicrobial properties. However, these candidate materials have to be tested for their antimicrobial efficacy in sophisticated screening procedures that mostly include microscopic examination. For increased specificity and sensitivity, microorganisms are usually stained with fluorescent dyes or probes. However, these staining procedures often require numerous washing steps whereby loosely bound biofilms can be easily washed away and thus impeding data analysis and interpretation. Furthermore, DNA-intercalating dyes are often carcinogen or at least toxic, making their handling and disposal more demanding. The aim of this study was therefore the establishment of a fast and easy method without complex staining procedures which can be used for initial screens. For this purpose we made use of a well-known property of aldehyde fixatives: the generation of fixative-induced fluorescence. This normally can be a problem if using specific probes or dyes because it complicates the distinction of real signals from the fixative-induced fluorescence in histological tissues [3]. During the fixation with glutaraldehyde, which was first described as a fixative for electron microscopy in 1963 [4], the aldehyde reacts with amino groups of proteins in the tissue via covalent cross-linking $[5,6]$. Bacteria contain a large number of membrane proteins which can react with the aldehydes during the fixation process. Their product, a Schiff base, leads to a high autofluorescence of the cells while microscopic examination $[7,8]$. The fixation procedure is easy to perform, cost efficient and increases the mechanical stability of biofilms [9-11]. We expected that a speciesspecific membrane protein composition would result in 


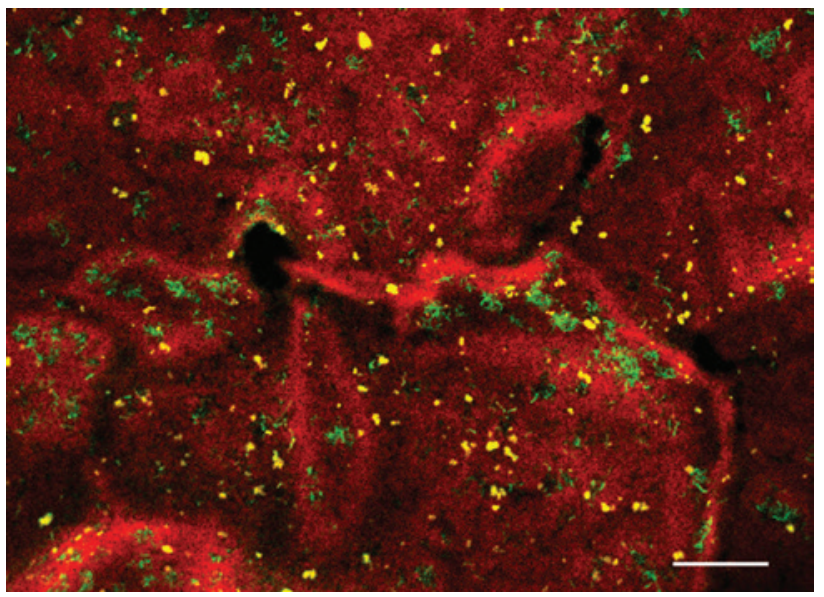

different fluorescence intensities. Initially, we performed a multiprobe fluorescence in situ hybridization assay (urea-NaCl-FISH; modified after [12]). Due to probe labeling with different fluorescent dyes, this method allowed the simultaneous localization of three individual bacterial species within a biofilm. To analyze the biofilm with species-specific labels, confocal laser scanning microscopy (CLSM; C2si, NIKON, Tokio, Japan) was used. The urea-NaCl-FISH experiment (Figure 1) showed Streptococcus oralis as the most prevalent species (red). Actinomyces naeslundii occurred in bigger aggregates (green) whereas Veillonella dispar formed occasional spots or little microcolonies (yellow).

With this background knowledge, we started NSLM analysis of autofluorescence signals. Therefore, the
Figure 1: Exemplary CLSM image of a biofilm stained with urea-NaCl-FISH.

The biofilm contains the bacterial species S. oralis (Probe MIT-588 [13]; labeled with Alexa-405; red), A. naeslundii (Probe ANA-103 [13]; labeled with Alexa-488; green) and V. dispar (Probe VEI-217 [14]; labeled with Alexa-568; yellow). S. oralis dominates the biofilm, whereby $A$. naeslundii occurs as bigger aggregates and $V$. dispar as single cells or microcolonies. FISH was chosen in order to correlate the single species arrangement within the biofilm with the NLSM results of fixative-induced fluorescence. S. oralis ATCC 9811 was acquired from the American Type Culture Collection (ATCC); $A$. naeslundii DSM 43013 and V. dispar DSM 20735 from the German Collection of Microorganisms and Cell Cultures (DSMZ). The bacteria were precultured under anaerobic conditions $\left(80 \% \mathrm{~N}_{2}, 10 \% \mathrm{H}_{2}, 10 \%\right.$ $\mathrm{CO}_{2}$ ) in brain heart infusion medium (BHl; Oxoid, Wesel, Germany) supplemented with $10 \mu \mathrm{g} / \mathrm{mL}$ vitamin $\mathrm{K}$ at $37^{\circ} \mathrm{C}$. Subsequent to the biofilm experiments, each planktonic preculture was adjusted to an optical density at $600 \mathrm{~nm}\left(\mathrm{OD}_{600}\right)$ of 0.1 . To obtain a threespecies biofilm, $300 \mu \mathrm{L}$ of $S$. oralis, $300 \mu \mathrm{L}$ of $A$. naeslundii and $300 \mu \mathrm{L}$ of $V$. dispar were mixed with $2.1 \mathrm{~mL} \mathrm{BHI/vitamin} \mathrm{K} \mathrm{medium.}$ From these mixtures, $150 \mu \mathrm{L}$ were applied to individual wells of a 12-well $\mu$-Chamber Slide (ibidi, Martinsried, Germany) and grown for $48 \mathrm{~h}$ under anaerobic conditions $\left(80 \% \mathrm{~N}_{2}, 10 \% \mathrm{H}_{2}, 10 \% \mathrm{CO}_{2}\right)$ at $37^{\circ} \mathrm{C}$. Urea-NaCl-FISH was modified after [12]. Biofilm images were acquired with a confocal laser scanning microscope (CLSM) (C2si, NIKON, Tokio, Japan). The image was edited with ImageJ [15]. Scale bar: $30 \mu \mathrm{m}$.

laser scanning microscope TriM Scope II (LaVision Biotec $\mathrm{GmbH}$, Bielefeld, Germany) equipped with the titanium:sapphire femtosecond laser system Chameleon Ultra II (Coherent Inc., Santa Clara, CA, USA) was used. With such a microscope short laser pulses from the
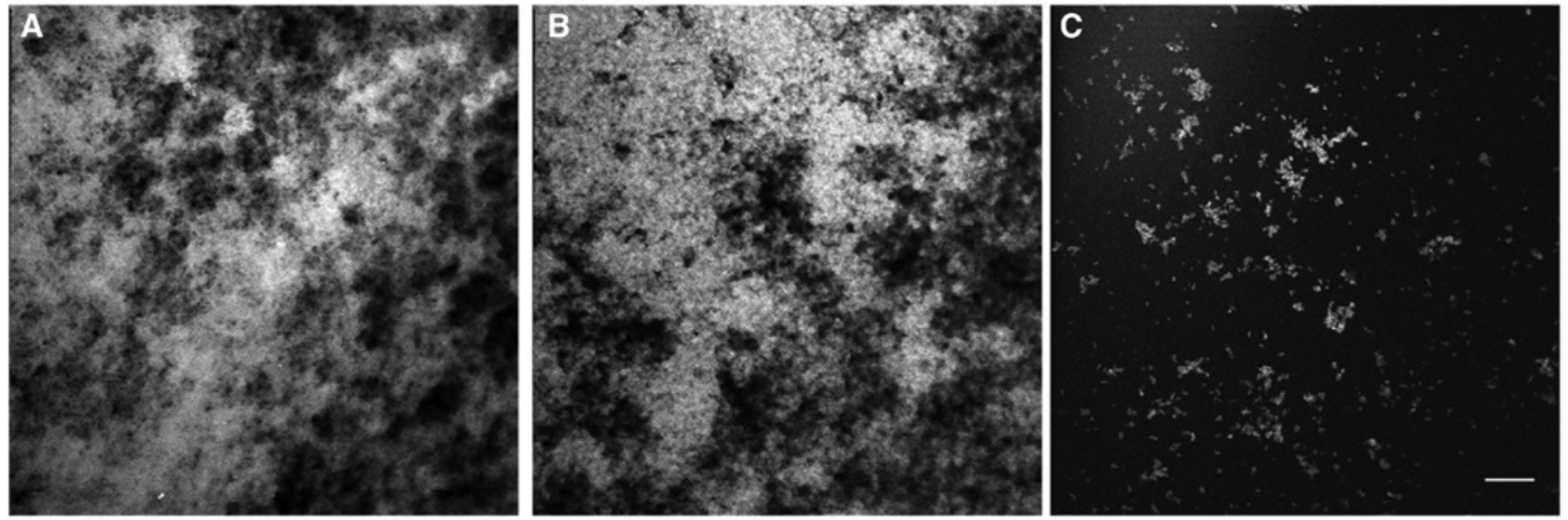

Figure 2: Exemplary NLSM images of $48 \mathrm{~h}$ old biofilms of (A) S. oralis, (B) A. naeslundii, and (C) V. dispar.

For the development of single-species biofilms, $300 \mu \mathrm{L}$ of each species suspension were mixed with $2.7 \mathrm{~mL}$ fresh BHI/vitamin $\mathrm{K}$ medium to acquire a final $\mathrm{OD}_{600}$ of 0.01 . The subsequent cultivation was carried out as described before. After $48 \mathrm{~h}$, the biofilms were fixed for 30 min using 2.5\% glutaraldehyde (Roth, Karlsruhe, Germany) before the silicone cultivation chamber was removed. The glass slides were covered with Dulbecco's Phosphate Buffered Saline (PBS; Biochrom GmbH, Berlin, Germany) and a glass coverslip (ibidi, Martinsried, Germany) before microscopic examination. The experiments were prepared in triplicate. The images were edited with ImageJ [15]), Every image shows a representative imaging plane inside a biofilm. Scale bar: $30 \mu \mathrm{m}$. 
near infrared are focused into the sample. Simultaneous absorption of two-photons within the focal volume excites fluorescent molecules inside the biofilm. After internal molecular conversion processes a transition from the excited state into the ground state finally leads to fluorescence. Since the excitation is restricted to the focal volume no pinhole (as in confocal microscopy) is necessary in the detection beam paths. Thus, spatially localized excitation takes place and defined imaging planes can be scanned. In the experiments excitation of two-photon fluorescence was realized with a central laser pulse wavelength of $780 \mathrm{~nm}$. In all cases, two-photon autofluorescence signals could be observed over a broad wavelength range (435$485 \mathrm{~nm}$ and 500-550 $\mathrm{nm}$; detectable wavelength regimes
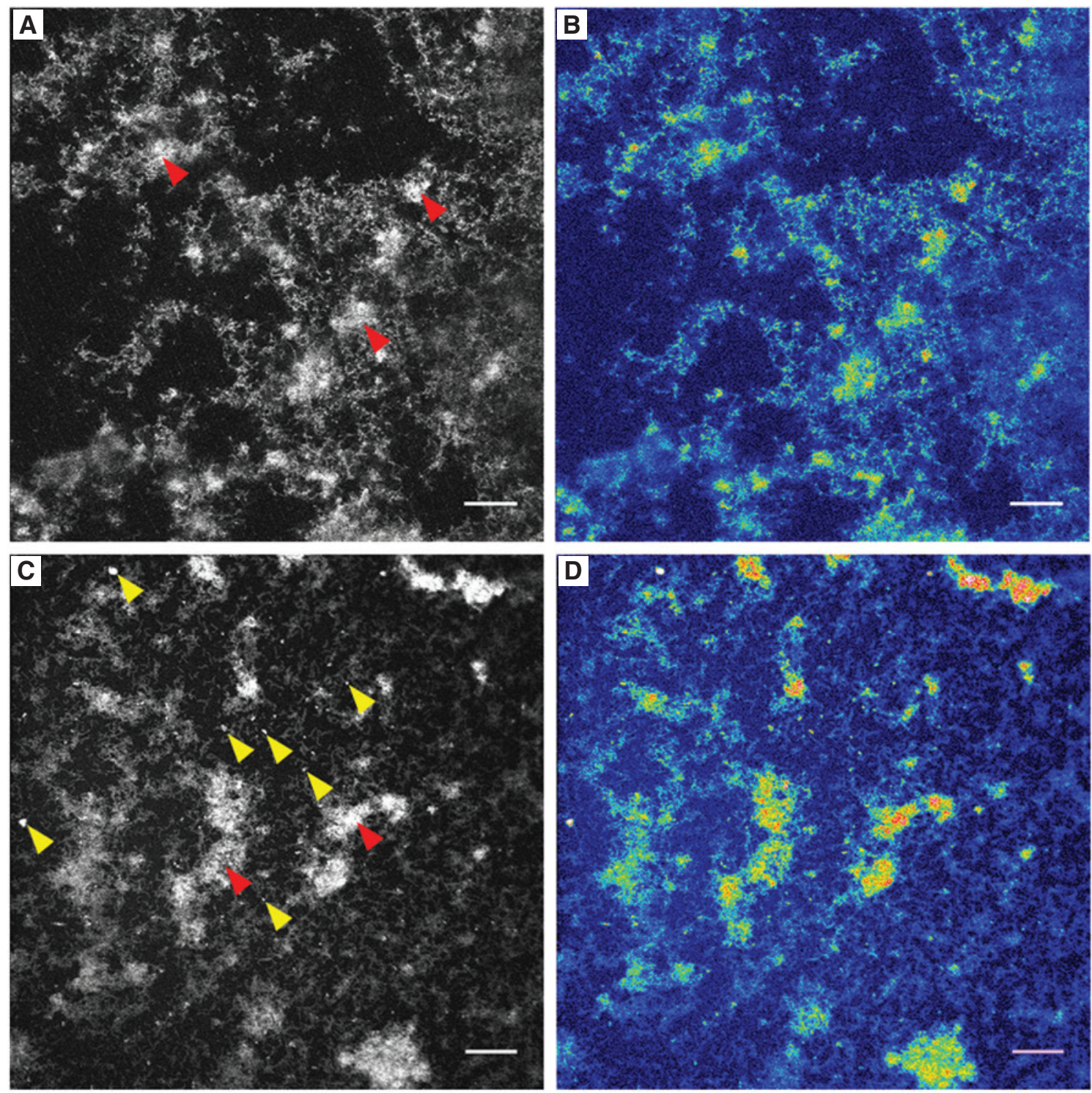

Figure 3: Exemplary NLSM images of $48 \mathrm{~h}$ old biofilms of (A) a two-species biofilm containing S. oralis and $A$. naeslundii and (B) a threespecies biofilm containing $S$. oralis, $A$. naeslundii and $V$. dispar.

To obtain a two-species biofilm, $300 \mu \mathrm{L}$ of $S$. oralis and $300 \mu \mathrm{L}$ of $A$. naeslundii preculture $\left(\mathrm{OD}_{600}=0.1\right)$ were mixed together with $2.4 \mathrm{~mL}$ fresh BHI/vitamin K medium. To obtain a three-species biofilm, $300 \mu \mathrm{L}$ of S. oralis, $300 \mu \mathrm{L}$ of $A$. naeslundii and $300 \mu \mathrm{L}$ of $V$. dispar were mixed with $2.1 \mathrm{~mL} \mathrm{BHI} /$ vitamin $\mathrm{K}$ medium. Cultivation, fixation and microscopic examination were carried out as described before. Image pairs (A), (B) and (C), (D) show identical biofilm sections, both in grayscale (left) and pseudocolor (right; 16-color lookup table, Image); [15]). Large aggregates with a brighter fluorescence signal can be seen in the two-species biofilm in (A), exemplarily marked with red arrows.

These aggregates can also be seen in the three-species-biofilm in (C). In (B) and (D) these aggregates can be seen as green, yellow and red clusters. Additionally, within the three-species biofilm, brighter smaller spots or microcolonies can be seen in between, in (C) exemplarily marked with yellow arrows and in (D) as green, yellow and red spots. Scale bars: $30 \mu \mathrm{m}$. 

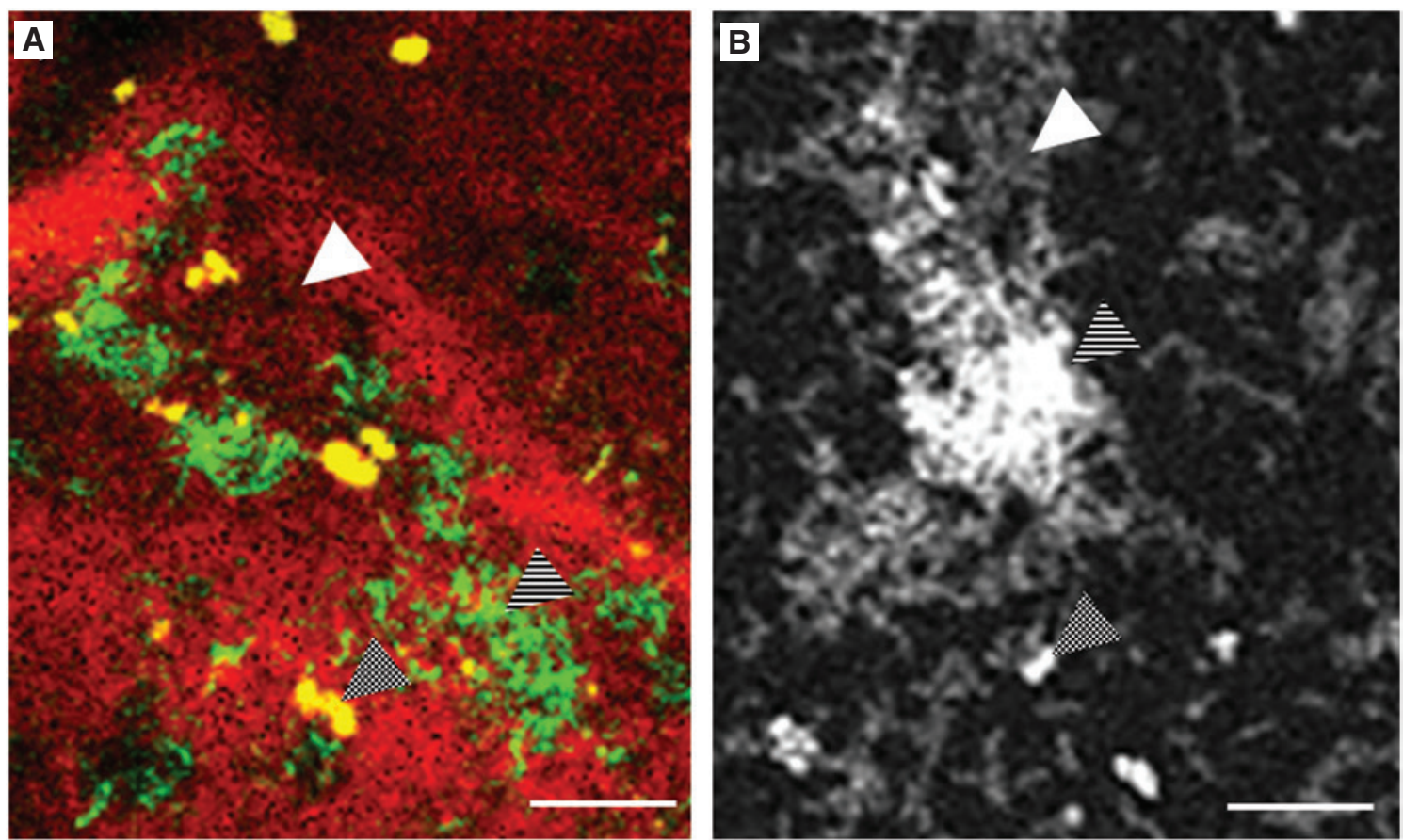

Figure 4: Comparison of urea-NaCl-FISH results (A) with autofluorecence image (B) of $48 \mathrm{~h}$ old three-species biofilm containing S. oralis, $A$. naeslundii and $V$. dispar.

The urea-NaCl-FISH experiment (A) demonstrated that the largest part of the biofilm shown in (B) belongs to S. oralis (white arrow). The brighter regions can be correlated to the aggregates of $A$. naeslundii (striped arrow) and the bright spots to $V$. dispar (pointed arrow). Scale bars: $10 \mu \mathrm{m}$.

defined by bandpass filters). The images from the two wavelength ranges were comparable and showed no difference in structural information. Therefore, only NLSM images on two-photon fluorescence signals in the range of 500-550 $\mathrm{nm}$ are displayed in this letter.

In a first step, single-species biofilms of $S$. oralis, $A$. naeslundii and $V$. dispar were investigated for their fixative-induced two-photon fluorescence patterns and are displayed in Figure 2. The $48 \mathrm{~h}$ old biofilms of $S$. oralis (Figure 2A) and $A$. naeslundii (Figure 2B) showed very dense and spongy structures compared to the thinner biofilm of $V$. dispar (Figure $2 \mathrm{C}$ ). Inside the single species biofilms, no differences regarding the fluorescence intensities could be detected. In contrast, brighter fluorescing aggregations of bacteria could be detected within a dualspecies biofilm in Figure $3 \mathrm{~A}$ and in a three-species biofilm in Figure 3C (in both exemplarily marked with red arrows). The aggregates can also be seen in the images which were modified with a 16-color lookup table as green, yellow and red cluster (Figure 3B, D). In the three-species biofilm the third bacterial species occurs occasionally as brighter spots between the two others (Figure 3C; exemplarily marked with yellow arrows). We were able to correlate these twophotone autofluorescence images from NLSM with the results from urea-NaCl-FISH (Figure 1) and demonstrated the similarities in Figure 4: S. oralis dominates the biofilm (exemplarily marked with a white arrow). The brighter fluorescing aggregates could be identified as A. naeslundii (exemplarily marked with a striped arrow) and the spots or microcolonies could be identified as $V$. dispar (exemplarily marked with a pointed arrow). We were able to distinguish between the three bacterial species based on different twophoton autofluorescence intensities.

In situ hybridization labeling is more laborious and costly than the glutaraldehyde fixation method. The latter is therefore more suited for standardized medium to high volume screening applications where cost-effectiveness and ease of use are given priority. A further advantage is the direct fixation of the biofilms without previous staining and washing procedures. We think that this method, once a biofilm-model is established, can be applied for the initial microscopic screening of new implant materials to get a first impression of their antimicrobial efficacy.

Acknowledgments: This work was carried out as an integral part of the BIOFABRICATION FOR NIFE Initiative, which is financially supported by the ministry of Lower Saxony and the VolkswagenStiftung. NIFE is the Lower Saxony Center for Biomedical Engineering, Implant Research and Development, a joint translational research 
centre of the Hannover Medical School, the Leibniz University Hannover, the University of Veterinary Medicine Hannover and the Laser Zentrum Hannover e. V.

Funding: Ministry of Lower Saxony and VolkswagenStiftung (both BIOFABRICATION FOR NIFE: VWZN2860).

\section{References}

1. Mombelli A, van Oosten MA, Schurch E, Jr, Land NP. The microbiota associated with successful or failing osseointegrated titanium implants. Oral Microbiol Immunol. 1987;2:145-51.

2. Mombelli A, Decaillet F. The characteristics of biofilms in periimplant disease. J Clin Periodontol. 2011;38:203-13.

3. Del Castillo P, Llorente AR, Stockert JC. Influence of fixation, exciting light and section thickness on the primary fluorescence of samples for microfluorometric analysis. Basic Appl Histochem. 1989;33:251-7.

4. Sabatini DD, Bensch K, Barrnett RJ. Cytochemistry and electron microscopy. The preservation of cellular ultrastructure and enzymatic activity by aldehyde fixation. J Cell Biol. 1963;17:19-58.

5. Bowes JH, Cater CW. The interaction of aldehydes with collagen. Biochim Biophys Acta. 1968;168:341-52.

6. Richards FM, Knowles JR. Glutaraldehyde as a protein crosslinking reagent. J Mol Biol. 1968;37:231-3.
7. Baschong W, Suetterlin R, Lang RH. Control of autofluorescence of archival formaldehyde-fixed, paraffin-embedded tissue in confocal laser scanning microscopy (CLSM). J Histochem Cytochem. 2001;49:1565-72.

8. Haney SA, Bowman D, Chakravarty A. An introduction to high content screening: imaging technology, assay development, and data analysis in biology and drug discovery. Hoboken, NJ: Willey; 2015.

9. Takeo Y, Oie S, Kamiya A, Konishi H, Nakazawa T. Efficacy of disinfectants against biofilm cells of Pseudomonas aeruginosa. Microbios. 1994;79:19-26.

10. Midelet G, Carpentier B. Impact of cleaning and disinfection agents on biofilm structure and on microbial transfer to a solid model food. J Appl Microbiol. 2004;97:262-70.

11. Simoes M, Pereira MO, Vieira MJ. Effect of mechanical stress on biofilms challenged by different chemicals. Water Res. 2005;39:5142-52.

12. Lawson TS, Connally RE, Vemulpad S, Piper JA. Dimethyl formamide-free, urea- $\mathrm{NaCl}$ fluorescence in situ hybridization assay for Staphylococcus aureus. Lett Appl Microbiol. 2012;54:263-6.

13. Thurnheer T, Gmur R, Guggenheim B. Multiplex FISH analysis of a six-species bacterial biofilm. J Microbiol Methods. 2004;56: 37-47.

14. Paster BJ, Bartoszyk IM, Dewhirst FE. Identification of oral streptococci using PCR-based, reverse-capture, checkerboard hybridization. Methods Cell Sci. 1998;20:223-31.

15. Schneider CA, Rasband WS, Eliceiri KW. NIH Image to ImageJ: 25 years of image analysis. Nat Meth. 2012;9:671-5. 